Agricultrices et diversification agricole : l'empowerment pour comprendre l'évolution des rapports de pouvoir sur les exploitations en France et aux Etats-Unis

Alexis Annes* and Wynne Wright

*Enseignant Chercheur

UMR LISST - Dynamiques Rurales,

INP-PURPAN, Toulouse, France

75 voie du TOEC

BP 57611

31076 Toulouse Cedex 3, France

Tel: (33) 561153086

Email: alexis.annes@purpan.fr

Associate Professor

Depts. of Community Sustainability and Sociology

131 Natural Resources Building

480 Wilson Road

Michigan State University

East Lansing, MI 48824, USA

Tel: (517) 884-1372

Email: wrightwy@anr.msu.edu

*Corresponding author 


\section{Agricultrices et diversification agricole : l'empowerment pour comprendre l'évolution des rapports de pouvoir sur les exploitations en France et aux Etats-Unis}

En France et aux Etats-Unis, les derniers recensements agricoles suggèrent une représentation croissante des femmes. En 2012, le nombre d'agricultrices américaines atteignait 969672, en France, elles étaient plus de 130000, soit dans les deux cas, 30\% de la population agricole ${ }^{1}$. Ces chiffres ne traduisent pas nécessairement une participation croissante des femmes à l'agriculture, mais suggèrent une visibilité plus importante contrastant fortement avec des décennies d'invisibilité. Comme l'ont suggéré plusieurs auteur-e-s (Barthez 2005 ; Brasier et al. 2014 ; Nicourt 2013 ; Sachs 1983), l'implication des femmes dans la production agricole, parce qu'elle avait lieu au sein de la famille, est longtemps restée inaperçue. Aussi bien en France qu'aux Etats-Unis, des travaux ont montré que les femmes ont contribué de façon significative à la réussite économique et au bien-être des exploitations (Lagrave 1996 ; Rosenfeld 1985). Cependant, leurs efforts sont souvent restés méconnus ou amoindris. De plus, dans ces deux contextes, l'expérience des femmes en agriculture s'est longtemps caractérisée par un statut social plus faible, un accès limité aux ressources matérielles, un dénigrement de leur identité professionnelle (Delphy 1983 ; Jellison 1993), bref, des rapports de pouvoir très inégalitaires. Néanmoins, les travaux les plus récents s'intéressant aux rôles des agricultrices dans les transformations des mondes agricoles, soulignent leur rôle clé. Ces travaux montrent qu'aujourd'hui les femmes sont plus souvent impliquées dans des démarches alternatives et innovantes (Jarosz 2011), ou apportent une nouvelle vision de l'agriculture et de nouvelles pratiques (Giraud and Rémy, 2013). Recensements agricoles américains et français montrent d'ailleurs bien que les agricultrices sont plus souvent impliquées dans les circuits courts de distribution, l'agriculture biologique, les activités de loisirs à la ferme ou d'hébergements touristiques. Elles sont également plus à même d'être à l'initiative de marchés de proximité (Agreste, 2012 ; USDA, 2014). Globalement, elles sont plus souvent impliquées dans des activités de diversification agricoles ${ }^{2}$.

La visibilité qui découle de cette plus forte implication nous offre la possibilité d'interroger l'évolution de l'expérience des femmes en agriculture et des rapports de pouvoir

\footnotetext{
${ }^{1}$ En France, les femmes sont plus représentées dans les exploitations spécialisées en élevage ovin/caprin, maraîchage/horticulture, et viticulture (Agreste, 2012) ; aux Etats Unis, dans les exploitations d'élevage (bovin, ovin, caprin et équin) et en polyculture (USDA, 2014).

${ }^{2}$ Nous entendons l'ensemble des activités allant au-delà de la « seule » production. Cela peut se traduire par une implication dans les circuits courts de distribution, de la transformation, des activités d'agritourisme et d'accueil à la ferme, ou encore l'engagement dans des signes officiels de qualité.
} 
sur les exploitations. Afin de répondre à cet objectif, nous utilisons le concept d'empowerment. L'utilisation ce concept est absent des travaux français sur le genre et l'agriculture. Inversement, dans le contexte nord-américain, plusieurs travaux ont souvent questionné dans quelle mesure certains choix stratégiques ont contribué à l'empowerment des agricultrices. Cependant, ils ne le définissent jamais réellement. Par exemple, dans leur étude des $\mathrm{AMAP}^{3} \mathrm{~s}$, DeLind et Ferguson (1999) se basent considérablement sur le processus d'empowerment pour expliquer les motivations des agricultrices à joindre ces organisations, mais elles ne proposent pas de définition du terme. Trauger (2004) se propose d'analyser comment l'agriculture durable fournit un contexte dans lequel les femmes peuvent s'exprimer plus librement. Si elle se demande dans quelle mesure ce type d'agriculture crée les conditions pour l'empowerment des femmes, elle ne le définit pas non plus. Pour trouver une opérationnalisation du concept, il faut se tourner vers les travaux réalisés par les spécialistes du développement agricole dans les pays $\mathrm{du}$ Sud. Ces chercheuses considèrent l'empowerment comme à la fois un processus et une capacité d'agir. Elles (voir par exemple Charlier 2006) le problématise en s'intéressant au concept de pouvoir et en identifiant quatre sous-types : le «pouvoir sur », le «pouvoir de », le « pouvoir avec», et le «pouvoir intérieur ». L' «empowerment» devient alors tributaire de l'acquisition des trois derniers types de pouvoir. Cette opérationnalisation nous permet de souligner le caractère dynamique de l'empowerment, ainsi que la complexité et la nonuniformité de son processus (l'acquisition d'un type de pouvoir ne signifiant pas nécessairement l'acquisition des autres). Dans le reste de cette contribution, nous discutons comment l'implication dans des activités de diversification permet aux agricultrices d'acquérir « pouvoir de », «pouvoir avec » et «pouvoir intérieur».

Nous nous appuyons sur un travail de recherche réalisé dans deux contextes culturels différents : le Sud-ouest français et le Midwest américain (l'état du Michigan plus particulièrement) ${ }^{4}$. Travailler sur deux contextes culturels différents nous permet de mettre en évidence de façon plus complète comment le concept d'empowerment permet de rendre compte de l'évolution des rapports de pouvoir et de l'expérience des femmes dans les exploitations

\footnotetext{
${ }^{3}$ Association pour le Maintien d'une Agriculture Paysanne.

${ }^{4}$ Les données que nous présentons ici sont issues d'un projet de recherche plus large cherchant à comparer les expériences et trajectoires de vie des femmes impliquées dans les agricultures étasunienne et française. Ce projet a été financé par une bourse de Soutien à la Mobilité Internationale de l'Institut National Polytechnique de Toulouse.
} 
agricoles $^{5}$. Entre 2012 et 2014, nous y avons rencontré ${ }^{6} 62$ agricultrices (30 en France, 32 aux Etats-Unis) avec une variété d'expériences et de parcours de vie. Cette diversité se traduit par des participantes qui étaient célibataires ou en couple, pratiquaient l'agriculture seules ou avec une autre personne (principalement leur conjoint ou leurs enfants) ou bien avaient choisi l'agriculture comme première carrière ou comme seconde. La très large majorité avait un statut officiel sur l'exploitation (que ce soit cheffe d'exploitation, co-exploitante ou salariée). Seule une agricultrice française et deux américaines n'avaient pas de statut officiel ${ }^{7}$. La taille des exploitations variait, de 0.2 ha à 208ha en France, et de 0.4ha à 220ha aux Etats Unis, et un peu plus de la moitié des enquêtées étaient engagées dans des pratiques d'agriculture biologique. Productions végétales (maraichage, horticulture et vigne) et productions animales (ovin, caprin, volaille et apiculture) étaient représentées. Si la plupart des agricultrices rencontrées étaient impliquées dans des activités reflétant les productions dominantes de chaque région, une minorité était impliquée dans des activités dite « de niche » (par exemple production de savon au lait d'ânesse en France ou production de thé ou de lavande dans le Michigan). Pour finir, si l'ensemble des agricultrices françaises peuvent vivre de leur activité, un peu moins d'un tiers des participantes américaines reconnaissent dépendre des revenus de leur époux.

L'ensemble des participantes ont néanmoins en commun leur implication dans des activités de diversification. Il s'agissait de transformation d'une production végétale (en confiture, sorbet, tisane ou sirop), animale (canard gras ou charcuterie) ou d'origine animale (fromage, yaourt, miel), de vente directe (à la ferme, sur un marché de plein vent, ou dans un magasin de producteurs), d'accueil à la ferme et agritourisme (ferme pédagogique, accueil social, gîtes, chambres et tables d'hôtes), ou encore d'une production sous signe officiel de qualité. Considérant la taille de notre échantillon, il est important de souligner que nos résultats

\footnotetext{
${ }^{5}$ En travaillant sur deux contextes culturels différents notre objectif n'est pas tant de comparer ces deux contextes que d'observer un éventail plus large de comportements et ainsi d'améliorer notre compréhension du processus d'empowerment chez les agricultrices.

${ }^{6}$ Lors de chaque rencontre nous avons conduit des entretiens compréhensifs suivis de la visite de l'exploitation.

${ }^{7}$ Parmi les agricultrices françaises, 13 gèrent seules l'exploitation agricole, onze sont co-exploitantes, et, en plus d'être en charge de l'activité de diversification, partagent le reste des tâches liées au fonctionnement de l'exploitation avec leur partenaire (mari, fils ou fille). Six sont uniquement en charge de l'activité de diversification sur l'exploitation et ne participent pas nécessairement aux décisions liées au fonctionnement global de l'exploitation. Parmi ces six, une n'a pas de statut officiel. Parmi les agricultrices américaines, 21 gèrent seules l'exploitation, deux sont co-exploitantes et, en plus d'être en charge de l'activité de diversification, partagent le reste des tâches liées au fonctionnement de l'exploitation avec leur partenaire. Neuf sont uniquement en charge de l'activité de diversification sur l'exploitation et ne participent pas nécessairement aux décisions liées au fonctionnement global de l'exploitation (parmi ces neufs, deux n'ont pas de statuts).
} 
ne reflètent pas l'expérience de l'ensemble des femmes en agriculture (en particulier celles qui ne sont pas impliquées dans des activités de diversification ou qui n'ont pas de statut officiel sur l'exploitation). Avec ces données, nous initions une réflexion sur l'évolution des rapports de pouvoir dans le cadre de l'implication des femmes dans de nouvelles formes d'agriculture telles que les activités de diversification.

\section{Les transformations des agricultures française et étasunienne au prisme du genre}

En France et aux Etats-Unis, les expériences des agricultrices suivent des chemins similaires. Les travaux existants révèlent que la subordination des femmes est ancrée historiquement et culturellement. Ainsi, l'invisibilité des agricultrices a été accomplie au travers de moyens socio-culturels et juridiques. Elle trouve son origine dans l'organisation des sociétés paysannes en France (Barthez, 1982) et agrariennes aux Etats-Unis (Sachs, 1983) dans lesquelles elles étaient d'abord sous l'autorité du père, ensuite celui de leur époux et de sa lignée. A partir des années 50, un idéal modernisateur s'est imposé dans les deux pays. Le développement d'une agriculture moderne a profondément modifié l'organisation sociale des campagnes. Dans cet idéal, qu'il s'agisse de l'exploitation à 2 UTH en France (deux unités travail homme, c'est-à-dire une exploitation nécessitant deux temps pleins) ou de la «family farm » aux Etats-Unis, le couple hétérosexuel devient le socle d'une agriculture spécialisée et intensive. Ce nouveau modèle s'oppose alors à celui pluri-générationnel caractérisant les sociétés paysannes et agraires, désormais perçu comme passéiste. Si dans les discours, les femmes apparaissent comme des associées, dans la pratique, elles ne peuvent se réaliser qu'en tant qu'épouse ou mère : aux hommes la sphère professionnelle et le statut de chef d'exploitation, aux femmes la sphère domestique, le statut de mère et/ou d'épouse et l'invisibilité. Dans les deux contextes culturels, la modernisation marque donc l'éloignement des femmes de l'activité de production pour être confinées à la sphère domestique (Bessière 2004 ; Brasier et al. 2014). Certaines ont d'ailleurs trouvé un travail en dehors de l'exploitation. En faisant disparaître les lieux de socialisation féminins, la modernisation des campagnes a également accentué l'isolement des femmes, en particulier celles qui sont restées sur les exploitations (Cleary 1989 ; Granié et Guétat 2006 ; Sachs 1983). Isolement et solitude sont devenus des aspects critiques de leur expérience.

A partir des années 80 , dans les deux pays, une remise en question du modèle agricole productiviste émerge. Une agriculture «multifonctionnelle » associant au rôle de production ceux de valorisation du patrimoine, préservation de l'environnement ou encore de maintien du tissu social, est encouragée par les pouvoirs publics. Des activités dites de diversification 
agricole, telles que la transformation, la vente directe ou l'accueil, ajoutant de la valeur à la seule production de matière première et générant des revenus supplémentaires, sont encouragées et reconnues. Cet accent nouveau sur une agriculture plus diversifiée et surtout sur des activités non nécessairement en lien direct avec la production agricole a donné aux femmes l'occasion de réinvestir le devant de la scène agricole. Les derniers recensements agricoles et les travaux académiques montrent que les femmes sont plus à même d'être impliquées dans ces activités de diversification. Les tâches réalisées dans ce cadre s'appuient sur des compétences et font appel à des savoirs et subjectivités perçues et socialement construites comme féminines. Différents travaux suggèrent que ces activités permettent aux agricultrices de valoriser et d'utiliser leur savoir-faire en matière de soin, service et éducation (Guetat et al. 2011). Ainsi, les agricultrices peuvent passer d'une position d'invisibilité sociale à des rôles reconnus et prometteurs pour leur «empowerment » personnel.

\section{« Pouvoir de » pratiquer l'agriculture}

Dans un premier temps, nous nous intéressons au premier aspect de l'empowerment, le « pouvoir de ». Nous interrogeons comment l'implication dans des activités de diversification agricole favorise la capacité des agricultrices à prendre des décisions, se fixer des objectifs, et exercer un libre arbitre.

Tout d'abord, en choisissant d'être agricultrices, participantes françaises et américaines expriment un «pouvoir de » réaliser leurs propres objectifs de carrière et d'exercer un libre arbitre. En effet, la majorité d'entre elles ont choisi d'être agricultrice. Si, historiquement en France (Barhez, 2005) et aux Etats Unis (Sachs, 1983), l'entrée dans le métier se faisait par adoption de la profession du conjoint, ce n'est plus automatiquement le cas dans nos échantillons. Par exemple, parmi les participantes américaines, une large majorité est devenue agricultrice indépendamment du travail ou de la volonté du mari. Elles ont choisi l'agriculture. La plupart d'entre elles (21 sur 32) dirige leur activité seule soit parce qu'elles ne sont pas mariées, soit parce que leur mari travaille à l'extérieur. Ces derniers peuvent les aider de temps en temps, en particulier durant les périodes de travail les plus chargées, mais ils n'ont que très peu, pour ne pas dire aucune, responsabilité permanente sur l'exploitation ${ }^{8}$. Par rapport à

\footnotetext{
${ }^{8} \mathrm{Au}$ final, parmi les agricultrices américaines, 21 sont seules cheffes d'exploitation, deux sont co-exploitantes et, en plus d'être en charge de l'activité de diversification, partagent le reste des tâches liées au fonctionnement de l'exploitation avec leur partenaire, et neuf sont uniquement en charge de l'activité de diversification sur l'exploitation et ne participent pas nécessairement aux décisions liées au fonctionnement global de l'exploitation (parmi ces neufs, deux n'ont pas de statuts). Parmi les agricultrices françaises, 13 sont seules cheffes d'exploitation, onze sont co-exploitantes, et, en plus d'être en charge de l'activité de diversification, partagent le reste des tâches liées au fonctionnement de l'exploitation avec leur partenaire, et six sont uniquement en charge
} 
l'échantillon français, la très large majorité des agricultrices s'est également engagée dans l'agriculture de façon intentionnelle (24 sur 30). Parmi celles-ci, 13 ont choisi de devenir agricultrice indépendamment de la carrière de leur partenaire, soit parce qu'elles sont/étaient célibataires, soit parce que leur partenaire avait une autre profession. Pour d'autres (11), ce fut un choix de couple de s'installer en agriculture. Pour finir, l'ensemble des participantes se présentent comme les seules décideuses ou les décideuses principales, soit sur l'ensemble de l'activité ou sur une activité en particulier (comme la transformation par exemple).

Pour les femmes, comme pour les hommes, s'installer en agriculture est un chemin difficile à suivre. L'accès à la terre et au capital sont souvent des facteurs limitants, voir bloquants. Cependant, les sociologues ont montré l'existence de barrières spécifiques pour les femmes (Jacques-Jouvenot 1997 ; Rieux et Dahache 2008). Par exemple, en France, Rieux et Dahache (2008) ont montré que l'accès aux moyens de production était plus difficile pour les femmes du fait de la persistance d'une idéologie patriarcale. Sans surprise, les agricultrices américaines et françaises ont mis l'accent sur cette question de l'accès au foncier comme centrale à la poursuite de leur objectif de mener à bien une activité agricole. Ainsi, posséder un capital financier de départ ou hériter de l'exploitation agricole familiale, leur a donné le « pouvoir de » conduire leur activité plus facilement que d'autres agricultrices. Elles possèdent ainsi une exploitation agricole avec non seulement des terres, du foncier bâti, des productions végétales et/ou animales qui «fonctionnent», mais également avec une reconnaissance, une clientèle et un savoir-faire. Elles bénéficient donc d'un bon capital de départ pour ensuite modeler cette exploitation selon leurs objectifs, leurs envies, et leurs projets.

Les agricultrices américaines attribuent également la possibilité de mener à bien leur activité agricole à leur précédente carrière qui leur a permis de développer des compétences utiles à leur nouveau métier. Tenir des comptes, commercialiser des produits, en faire la publicité, créer un site internet, accueillir des touristes et expliquer son travail, transmettre des connaissances et s'occuper des autres, sont des activités qui faisaient parties de leur quotidien professionnel passé et qu'elles ont pu mettre en pratique. Par exemple, Leigh explique qu'elle utilise ses «compétences de professeurs des écoles tous les jours ». De diriger ses deux employés, à enseigner des cours de fabrication de pain, en passant par proposer une visite guidée de son ancien moulin à farine, elle précise qu'elle est en permanence en posture d'enseignement. Bien que ces compétences ne soient pas uniquement requises dans le cadre

de l'activité de diversification sur l'exploitation et ne participent pas nécessairement aux décisions liées au fonctionnement global de l'exploitation (parmi ces six, une n'a pas de statuts officiel). 
d'activités agricoles de diversification, elles deviennent clés pour des activités impliquant un contact avec un public.

Les agricultrices françaises n'ayant pas hérité une exploitation n'ont pu acheter que des petites propriétés agricoles ou simplement quelques hectares afin de démarrer leur activité 9 . S'engager immédiatement dans des activités de diversification leur a permis d'ajouter de la valeur à leur production de base, et ainsi générer un revenu sur de plus petites surfaces. C'est le cas d'Hélène qui explique : « comme on a une très petite surface, notre idée était d'avoir une production sur laquelle on pouvait ajouter de la valeur ». C'est pourquoi elle a décidé : «de produire des petits fruits et de les transformer sur place, en confiture, sirops, coulis, et d'autres trucs comme ça ». De plus, dans le cadre de ces activités de diversification, les agricultrices françaises insistent sur le fait qu'elles leur fournissent un espace où exprimer leur créativité et où trouver des solutions aux problèmes rencontrés par elles-mêmes. Expérimenter, créer de nouveaux produits, se tromper et recommencer caractérisent la pratique de ces agricultrices. Marion raconte comment, lors de sa première année en tant qu'agricultrice, alors qu'elle venait seulement de planter ses bulbes de safran et devait attendre l'année suivante avant la première récolte, elle a improvisé. A partir des ressources qu'elle possédait autour de sa ferme (fleurs de sureau, de tilleul, mais aussi menthe sauvage), elle a confectionné des sirops et des bonbons qu'elles vendaient sur les marchés. Ces résultats prolongent les conclusions de Guétat-Bernard et Pionetti (2016) qui suggèrent que «moins impliquées dans le processus de modernisation, [les femmes] ont été contraintes de redoubler de ruse, de pratiques de contournement/détournement pour maintenir leur place ». Ainsi, les agricultrices rencontrées se créer un espace au sein de l'activité agricole en capitalisant sur de nouvelles activités et en faisant preuve de créativité.

Interroger l'expérience des agricultrices au regard du « pouvoir de » qu'elles acquièrent en engageant des activités de diversification nous montrent donc qu'elles possèdent une capacité d'action. Cette possibilité de conduire leur projet est favorisée par différents facteurs (héritage familial, valorisation du capital humain, choix d'un mode de production) qui permettent de pérenniser l'activité et de concrétiser les objectifs de chacune. Cependant, l'existence de ces éléments ne doit pas cacher pour certaines agricultrices la persistance de freins à l'expression de leur capacité d'action. C'est le cas particulièrement pour deux

\footnotetext{
${ }^{9}$ Cela est le cas des hommes dans des situations similaires (manque de capitaux financiers et fonciers, et de capital social agricole), cependant, comme l'a montré Dahache (2010), du fait du point du référentiel masculin en agriculture, les femmes ont plus de difficultés à accéder au foncier (plus facilement vendu à un homme) ou à contracter des prêts bancaires (manque de ressources propres, banques émettant des réserves quant à la viabilité de leur projet).
} 
catégories d'agricultrices françaises : celles, plus jeunes, qui ont repris l'exploitation familiale et celles, plus âgées, qui se sont installées sur l'exploitation de leur mari et y ont développé une activité d'accueil touristique.

Les plus jeunes participantes françaises ont évoqué le manque de soutien de leurs parents à l'évocation de leur projet de reprise parce qu'elles sont des femmes. Pour leurs parents, l'agriculture demeure un métier d'homme. Par exemple, pour les parents de Sandrine, c'est leur fils qui aurait dû reprendre l'exploitation. Sandrine se souvient qu'au départ, ses parents étaient non seulement déçus que leur fils de reprennent pas la ferme familiale, mais ils n'envisageaient pas la possibilité qu'elle la reprenne. Pascale s'est vue retirer le soutien de son père lorsqu'elle s'est séparée de son conjoint et qu'elle s'est retrouvée seule pour mettre en place son projet de reprise de l'exploitation familiale. Elle explique : «il [son père] n'y croyait pas... une fille seule. Ça ne lui plaisait pas » et ajoute : «j'ai dû courber le dos la première année pour prouver que je pouvais le faire ». D'autres participantes ont évoqué des difficultés à être perçues comme les responsables de l'exploitation. En effet, aux yeux de leurs paires ou des client-e-s, les parents restent les chefs d'exploitation, leur fille ayant un rôle d'aide.

Les agricultrices les plus âgées de notre échantillon français, qui se sont installées sur l'exploitation de leur mari suite au départ à la retraite de leurs beaux-parents ont également une capacité d'agir limitée. A la suite de leur installation, elles ont créé une activité de diversification (de type accueil touristique) dont elles sont responsables et qui leur donne une légitimité. Si elles sont assez libres de gérer leur activité comme bon leur semble, elles n'ont que très peu à dire sur le reste du fonctionnement de l'exploitation. De même, dans la gestion quotidienne de l'exploitation, leur activité demeure secondaire aux yeux de leur époux et du reste de la famille. Dans la lignée des travaux de Giraud (2011), nous avons montré ailleurs (Annes et Wright, 2015) que ces agricultrices se sont créés un espace de liberté et font preuve d'autonomie dans la conduite de leur activité, mais dans un espace de contrainte. Si leur activité vient à entrer en conflit avec les autres activités de l'exploitation, c'est la leur qui sera sacrifiée.

Pour finir, nous souhaitons aussi revenir sur le cas de certaines participantes américaines (9 d'entre elles) dont le mari participe à la santé financière de l'exploitation grâce à son travail en dehors de l'exploitation. Veronica, par exemple, explique que son «mari pourvoit grandement financièrement à [son] exploitation, ce qui l'« aide à [se] maintenir, car, après 3 ans, [elle] ne peu[t] toujours pas tirer un revenu ». Non seulement ces agricultrices n'ont pas les ressources requises pour maintenir durablement leur activité agricole, mais cette situation encourage une relation de co-dépendance des femmes envers les hommes qui peut s'interpréter comme un frein à l'acquisition de « pouvoir de ». 


\section{«Pouvoir avec » les consommateur.trice.s}

Ici, nous mobilisons la dimension « pouvoir avec » de l'empowerment. Cette dimension insiste sur l'importance d'appréhender l'empowerment comme une démarche collective. Des travaux précédents suggèrent que les agricultrices peuvent acquérir du pouvoir au travers des liens qu'elles établissent avec d'autres femmes au sein d'organisations agricoles ou de réseaux (voir par exemple Annes et Wright 2015). De la même manière, les agricultrices de notre étude relatent qu'une de leurs sources de motivation était le désir de contacts avec les autres. Ce faisant, mettre en place des activités agricoles de diversification prenait tout son sens. Ainsi, les agricultrices rencontrées s'impliquent dans des activités de diversification agricole comme moyen d'ancrer leur exploitation ainsi que leur personne dans un réseau social plus large. Charlotte (USA) met l'accent sur l'importance de la communauté quand elle dit: « c'était important pour nous de créer une exploitation qui serait ouverte au public pour que nous puissions partager notre espace avec d'autres personnes ». Leslie (USA) décrit son travail sur son exploitation spécialisée dans la production de menthe comme «semer du rêve, cultiver l'amitié et récolter de la bonté ». Grace (USA), elle aussi, pointe du doigt son désir de communauté quand elle dit: «ça ne m'intéresse pas de faire pousser du maïs ou du soja comme le font les agriculteurs autour d'ici. Je suis intéressée par les gens, j'ai besoin de parler aux gens ». Dans chacun de ces cas, un désir de créer des liens étroits avec des personnes en dehors de l'exploitation, de favoriser l'échange et le partage, apparaît. Ces interactions procurent aux agricultrices rencontrées une réelle satisfaction et leur permettent de nouer des liens étroits avec les client-e-s. Emilie (F) explique : « ce que j'aime à propos des marchés ? Le contact avec les gens ». Une autre agricultrice, Marie $(\mathrm{F})$, va plus loin en déclarant apporter un réel sens du service pour ses client-e-s : «ce que j'aime ce sont les clients. Tu les connais, tu commences à t'attacher et à connaître exactement leurs goûts ! » Plus tard dans l'entretien elle explique : «parfois, quand tu choisis tes produits et que tu charges ton camion, tu penses à ce qu'ils aiment, ce qui leur ferait plaisir ». Dans la lignée d'autres travaux (Guétat-Bernard et Pionetti 2016), ces résultats montrent que la relation avec les client-e-s transcende la relation commerciale et se transforme en un véritable souci de l'autre de la part des agricultrices. Nos données montrent également que les client-e-s développent un fort attachement pour les agricultrices. Anne-Marie (F), une productrice de fromage explique qu' «ils viennent acheter un morceau de la ferme ». Elle pense que sinon, «ils ne viendraient pas sur l'exploitation » car, dit-elle, «c'est une part de nous qu'ils achètent ». 
Au-delà de ces liens étroits, les agricultrices mettent également en avant leur rôle éducatif dans la relation aux clients et clientes. «Enseigner aux autres, c'est une de mes passions », précise Ava (USA). Elle rajoute : «je profite des moments que nous partageons ensemble pour parler de l'agriculture dans le Michigan, de l'opposition entre petites et grandes exploitations, et l'importance d'avoir des gens qui travaillent ensemble ». Sylvette (F), éleveuse de volailles biologiques profitent des interactions avec ses client-e-s pour leur expliquer la différence entre productions intensive et extensive, ainsi que les conséquences de ces mode de production sur la qualité du produit : «Mes clients me disent parfois que mes poulets sont durs. Je leur dis qu'ils ne sont pas durs, mais qu'ils sont fermes ! » Elle rajoute : «Un poulet de quatre mois n'est pas un poulet de deux mois avec les os qui se détachent ! Alors, je dois être présente, expliquer la différence ». L'ensemble des agricultrices, françaises et américaines, pensent que les gens ont besoin de plus d'éducation, et que c'est d'ailleurs «ce que nous faisons principalement » comme le précise Patricia (USA).

Nos résultats suggèrent donc que les activités de diversification agricole pourraient créer un contexte permettant de dépasser l'isolement qui a longtemps caractérisé l'expérience des femmes en agriculture en France et aux Etats Unis. Ces activités permettraient d'encourager de nouvelles formes de sociabilité entre agricultrices et consommateur.trice.s et remettrait en question la fragmentation du système agro-alimentaire en créant des relations de solidarité et de confiance entre populations agricoles et non-agricole. Ces nouvelles formes d'agriculture sembleraient remettre les relations sociales au centre de l'activité agricole. Cependant, il reste encore à évaluer dans quelle mesure ces liens exprimés par les agricultrices le sont aussi par les consommateur.trice.s.

\section{«Pouvoir intérieur»}

Si les agricultrices sont capables d'acquérir du « pouvoir de » et du «pouvoir avec » au travers de leur activité, dans quelles mesures gagnent-elles en «pouvoir intérieur»? Agricultrices françaises et américaines ont mis l'accent sur les bénéfices psycho-sociaux acquis suite à l'implication dans les activités de diversification et aux interactions sociales consécutives. Fréquemment, nous avons entendu des termes comme « agréable », « gratifiant » ou «épanouissant personnellement ». «Je pense que c'est un travail important, je suis douée pour ça. Ça me donne beaucoup de fierté de partager mon savoir, de voir que d'autres personnes prennent notre travail au sérieux » explique Angela (USA). Françoise (F) explique que pour elle «le contact humain et le bien-être de l'humain c'est plus important...c'est vraiment plus important qu'ils [les client.e.s] repartent avec le sourire que de faire une grosse vente. Un 
sourire, c'est gratifiant. » Pour certaines participantes, c'est l'aspect éducatif de leur travail qu'elles décrivent comme « un processus fascinant», "personnellement exaltant ». « Ça me fait me sentir bien d'éduquer les gens et de savoir que je les aide à se développer en tant que personne » précise Carolyn (USA). Pour d'autres participantes, c'est le fait de «produire de la nourriture » pour d'autres personnes qui est source de satisfaction. « Et puis je suis fière d'avoir donné mes produits à quelqu'un. C'est chouette quoi. J'aime produire des fromages que les gens achètent et apprécient » mentionne Marie (F). Ainsi, ces activités de diversification ont permis aux agricultrices d'acquérir un sentiment d'accomplissement dans leur travail. Elles possèdent une activité qui leur appartient et pour laquelle elles se sentent légitimes. Comme le résumé Véronique $(\mathrm{F})$, «c'est important d'avoir un projet. On se sent bien. D'ailleurs, c'est important pour tout être humain ».

Lors de nos rencontres avec les agricultrices, la question des difficultés liées au travail (isolement, pénibilité et surcharge) est parfois apparue, mais n'occupe pas une place centrale dans les discours. La pratique d'activité de diversification peut constituer un élément explicatif. Seules les agricultrices, qui dans un premier temps, n'avaient pas mis en place ces activités ont mentionné un sentiment d'isolement fort. La création d'activités d'accueil (tables et chambres d'hôtes ou visites de ferme) a permis de mettre un terme à ce sentiment. Pour les autres agricultrices, démarrage de l'activité de diversification et installation ont été concomitantes, ce qui peut expliquer que la question de l'isolement est peu revenue dans leur discours. Les questions de pénibilité et de surcharge du travail, quant à elles, ont été mentionnées de façon inégale par les participantes. Lorsqu'elles l'ont été, il s'agissait plus d'expliquer les moyens mis en place pour y remédier. Pénibilité et surcharge de travail semblaient donc moins subies que contrôlées. Par exemple, Pascale (F) explique que contrairement à son père, qui « s'épuisait complètement en période de taille des noyers », car «il voulait tout faire d'un coup », elle a décidé de travailler différemment en « [se faisant] un planning sur le mois ». « Je prends mon temps », dit-elle, « quitte à être un peu en retard sur la période de taille ».

L'implication dans les activités de diversification donne l'occasion aux agricultrices rencontrées de se construire une identité professionnelle agricole. Nos résultats confirment d'ailleurs les travaux les plus récents suggérant une tendance en hausse des femmes à revendiquer le statut professionnel d'agricultrice (Brasier et al. 2014). Dans l'échantillon américain, 8 participantes sur 10 s'auto-identifient comme agricultrices. Elles sont 9 sur 10 dans l'échantillon français. Insister sur cette revendication n'est pas anodin et cette visibilité accrue est loin d'être insignifiante. En tant que responsable d'activités de diversification, ces femmes ne sont plus reléguées au ban de l'agriculture, seulement considérées comme des aides 
familiales. Elles sont clairement visibles et capable d'utiliser ces activités pour se façonner une identité professionnelle et montrer aux yeux du public qu'elles possèdent un savoir spécifique. Cependant, si les agricultrices rencontrées revendiquent une identité professionnelle agricole, plusieurs d'entre elles reconnaissent avoir fait l'expérience de difficultés à être perçues de la sorte par les autres. Certains témoignages montrent que dans les consciences collectives, la représentation de la personne travaillant la terre est celle d'un homme. Ces représentations persistent aussi bien en France qu'aux Etats-Unis. Comme une agricultrice américaine l'explique, « les femmes ne sont pas vraiment considérées en agriculture. Avant, je faisais de la vente en gros et les clients ne m'adressaient pas la parole. Ils s'adressaient toujours à Brad [mari] ». Leslie (USA) confirme cette observation et se sent « agricultrice » mais, comme elle l'exprime, les autres la voit comme une « femme au foyer ». Du côté français, les agricultrices rencontrées ont également donné des exemples de situation où, dans le cadre professionnel, elles se sont senties invisibles et dénigrées par les hommes. Nadège, par exemple, se souvient d'une réunion syndicale où : «c'était juste comme si [elle] n'existai[t] ». Elle précise que «les gens ne [la] regardaient pas et ne [lui] parlaient pas » et conclue que « d'y être ou pas, ça aurait pas fait beaucoup de différence ». Certaines agricultrices françaises ont même décidé d'arrêter de se rendre à des réunions professionnelles. De façon assez paradoxale, si ces agricultrices subissent clairement la domination masculine (et peuvent apparaître freinées dans l'expression de leur «pouvoir de »), dans l'ensemble de notre échantillon, ce sont aussi les seules identifiant explicitement ces sources d'oppression (et faisant donc preuve d'un plus grand «pouvoir intérieur »).

\section{Activités de diversification : opportunité ou nouvelles contraintes ?}

Nos résultats suggèrent que les activités de diversification agricoles créent un contexte de redéfinition des rapports de pouvoir où les femmes sont plus visibles et font preuve d'une certaine capacité d'action. Dans le cadre de la conduite de leurs activités, le type de tâches et l'importance de compétences traditionnelles perçues comme féminine (comme cuisiner, vendre, accueillir, servir, etc.) leur donnent ainsi une position centrale et visible pour les cliente-s. Cependant, cette visibilité croissante ainsi que le recourt à des rôles, compétences et savoirs assignés au sexe des femmes, peuvent aussi indiquer des enjeux plus complexes. En effet, la visibilité des femmes dans le cadre d'activité de diversification pourrait signaler la persistance de leur invisibilité en tant que sujet. Quand les agricultrices travaillent avec leur conjoint (ou leur fils), nous observons la persistance d'une division traditionnelle du travail où les hommes exercent des activités d'extérieur et mécaniques, les femmes des tâches plus à même d'être 
réalisées à l'intérieur ou proche du foyer. Nos résultats montrent également que les femmes qui pratiquent l'agriculture seules (ou avec leur conjointe ou leur fille), ont tendance à être moins impliquées dans les travaux des champs ou mécaniques. La plupart d'entre elles ont développé des systèmes où peu de mécanisation est requise. Pour l'expliquer, agricultrices françaises et américaines mettent en avant plusieurs explications : manque de force physique des femmes, absence d'intérêt pour les machines ou encore désir de préserver sa « féminité ». La réticence à utiliser des machines nous donne une opportunité de questionner la persistance du «pouvoir sur ». Leurs explications peuvent s'inscrire dans un discours essentialiste qui positionne les femmes dans des rôles et des formes d'agriculture spécifiques. Elles peuvent aussi les empêcher de s'engager dans un domaine productif traditionnellement perçu comme masculin où production intensive et mécanisation sont des éléments de succès déterminants. Il est également possible que le rejet de la mécanisation témoigne de la résistance des agricultrices rencontrées à l'agriculture moderne et intensive. Dans ce cas-là, il ne s'agirait plus de persistance de «pouvoir sur» mais plutôt d'une façon d'exercer son libre arbitre et ainsi d'exprimer un « pouvoir de ». Cependant, nous ne pouvons pas écarter une autre interprétation. Considérant les difficultés auxquelles les femmes font face pour accéder au foncier et au capital, l'engagement dans des pratiques de diversification est peut-être moins idéologique que pratique. Avec moins de ressources financières, les femmes peuvent difficilement acheter du matériel agricole. Considérant les difficultés exprimées par les femmes lorsqu'elles souhaitent s'engager dans les organisations agricoles, la présence largement majoritaire d'hommes dans les CUMA $^{10}$ pourrait également être un frein à l'utilisation de cette solution alternative. Ces expériences concrètes peuvent conditionner le niveau de mécanisation.

Pour finir, nous souhaitons revenir sur la division traditionnelle du travail que nous avons observée. Celle-ci n'est pas problématique en elle-même, non qu'elle ne reflète nécessairement du « pouvoir sur ». Elle devient problématique, et symptomatique d'un contrôle et d'une domination, quand elle crée les conditions favorables au renforcement de logiques essentialistes qui assignent les femmes à des tâches spécifiques et immuables. Comme l'ont déjà suggérées Guetat-Bernard et al. (2011), rendre visible et mettre en scène pour la consommation touristique, rôles, compétences et savoirs assignés à leur sexe peut également s'interpréter comme une essentialisation du travail des femmes. Ainsi, à la place d'encourager leur empowerment, les activités de diversification agricole peuvent devenir coercitives et disciplinaires en créant pour les femmes les conditions d'adoption de rôles de genre

\footnotetext{
${ }^{10}$ Coopérative d'Utilisation du Matériel Agricole
} 
traditionnels. Les pratiques agricoles de diversification jouent de façon disproportionnée sur ces rôles, compétences, habituellement passés sous silence. Les rendre visibles est certes une étape importante du processus d'empowerment, mais peut également réduire les possibilités (et donc contraindre) des femmes à dévier de ces normes de genre. En ce sens, la visibilité nouvelle acquise par le biais de ces activités de diversification continue à perpétuer l'assignation des femmes à la sphère domestique.

\section{Conclusion}

A travers l'analyse de l'expérience d'agricultrices françaises et américaines impliquées dans des activités de diversification, nous souhaitions évaluer comment les rapports de pouvoir évoluent au sein des exploitations agricoles. L'étude des deux contextes culturels, nous montre de façon plus complète comment le concept d'empowerment permet de rendre compte de l'évolution des rapports de pouvoir. Cependant, il faut noter qu'à l'intérieur de chaque contexte culturel, le processus d'empowerment ne suit pas le même chemin. Qu'elles aient hérité de l'exploitation familiale ou qu'elles aient rejoint leur époux sur son exploitation, qu'elles évoluent dans des organisations professionnelles agricoles ou qu'elles choisissent de les éviter, des effets différenciés apparaissent. Nous insistons donc sur l'existence d'une diversité de rapports de pouvoir à l'intérieur même de ce groupe social que forment les agricultrices. Comme le suggère Calvès (2009), les travaux sur l'empowerment des femmes les ont trop souvent considérées comme catégorie unique et homogène. Différentes configurations et divers degré d'intensité caractérisent l'expression de l'oppression et de la domination (Hill-Collins 2000). Nos résultats montrent donc que les agricultrices n'ont pas toutes les mêmes expériences d'empowerment. Ainsi, les conditions et arrangements sociaux au sein desquels se déroulent ces activités de diversification agricoles doivent être interrogés plus en profondeur

Pour conclure, nous insisterons sur un point de vigilance quant à la pertinence de l'utilisation du concept d'empowerment tel que nous l'avons fait. Nous avons conclu que dans un contexte patriarcal qui amenuisait leurs capacités d'action, les femmes que nous avons interrogées, ont trouvé dans les activités de diversification agricole un moyen d'expression qu'elles n'avaient pas avant. Cependant, et de ce fait, nous supposons que les conditions de domination auxquelles elles étaient soumises sont restées les mêmes. Or, le patriarcat peut s'exprimer différemment et créer de nouvelles formes de contraintes pour les agricultrices. Ses modalités d'expression et ses contraintes peuvent avoir changé. Si le concept d'empowerment met en évidence les stratégies utilisées par les agricultrices pour agir et atteindre leurs objectifs, il ne permet pas de décrire avec précision la manière avec laquelle le patriarcat constitue un 
nouveau régime de pouvoir. Ainsi, sans vouloir nécessairement rentrer dans le débat sociologique sur ce que la sociologie anglo-saxonne appelle l'opposition « structure/agency », s'il nous semble que le concept d'empowerment nous permet de rendre compte des stratégies de résistance mises en place par les agricultrices, il peine à montrer comment les structures de pouvoir et d'oppression continue à agir sur les agricultrices.

\section{Liste des références citées}

Annes, A. et Wright, W. (2015). " 'Creating a Room of One's Own': French Farm Women, Farm Tourism and the Pursuit of Empowerment». Women Studies International Forum, 53.

Barthez, A. (2005). «Devenir agricultrice : À la frontière de la vie domestique et de la profession ». Economie Rurale, 289-290.

Brasier, K., C. Sachs, N. Kiernan, A. Trauger et M. Barbercheck. (2014). « Capturing the Multiple and Shifting Identities of Farm Women in the Northeastern United States ». Rural Sociology, 79, 3.

Calvès, A-E. (2009). «Empowerment : généalogie d'un concept clé du discours contemporain sur le développement ». Revue Tiers Monde, 200, 4.

Charlier, S. (2006). L'analyse de l'empowerment des femmes qui participent à une organisation de commerce équitable. Une proposition méthodologique. In C. Auroi et I. Yepez (Eds.), Economie Solidaire et commerce équitable. Acteur et actrices d'Europe et d'Amérique latine (pp. 87-110). Louvain, Presse Universitaires UCL/IUED.

Cleary, M. (2007). Peasants, politicians and producers: the organisation of French agriculture since 1918. Cambridge, Cambridge University Press.

Dahache, S. (2010). «La singularité des femmes chefs d'exploitation ». In : Hervieu, B., Mayer, M., Muller, P., Puseigle, F ; et J. Rémy (dirs.). Les mondes agricoles en politique. Paris, Les Presses de Sciences Po.

Delphy, C. (1983). «Agriculture et travail domestique : La réponse de la bergère à Engels ». Nouvelles Questions Féministes, 5, 3-17.

DeLind, L. and A. Ferguson. 1999. "Is This a Women's Movement? The Relationship of Gender to Community Supported Agriculture in Michigan ». Human Organization 58, 2.

Giraud, C. et Rémy, J. (2013). « Division conjugale du travail et légitimité professionnelle ». Travail, Genre et Société, 30.

Granié, A-M et H. Guétat-Bernard. 2006. Empreintes et inventivités des femmes dans le développement rural. Toulouse, Presse Universitaire du Mirail.

Guétat-Bernard, H, Granié, A-M et A. Terrieux (2011). «Initiatives féminines en agriculture et dynamiques des territoires ». In : G. Ferreol (ed.). Femmes et agricultures, pp.143-155, 2011, EME Editions Collection Proximités Sociologie.

Guétat-Bernard, H. et Pionetti, C. (2016). «Quand savoirs et imaginaire des agriculrices prêtent main-forte à la recherche agronomique : l'apport de la recherche-action ». In Devreux, A-M (éd.), Les sciences et le genre. Déjouer l'androcentrisme, Rennes, PUR.

Hill Collins, P. (2000). Black Feminist Thought: Knowledge, Consciousness and the Politics of Empowerment. New York, Routledge.

Jacques-Jouvenot, D. (1997). Choix du successeur et transmission patrimoniale. Paris, L'Harmattan. 
Jarosz, L. (2011). « Nourishing Women: Toward a Feminist Political Ecology of Community Supported Agriculture in the United States ». Gender, Place \& Culture, 18.

Jellison, K. (1993). Entitled to Power: Farm Women and Technology, 1913-1963. Chapel Hill, University of North Caroline Press.

Lagrave, R.M. (1996). Celles de la terre. Paris, EHESS.

Laisney, C. (2012). «Les femmes dans le monde agricole». Les Publications du Service des Statistiques et de la Prospective, Centre d'Etude et de Prospective, 38.

Nicourt, C. (2013). Etre agriculteur aujourd'hui: L'individualisation du travail agricole. Versailles, Edition QUAE.

Pilgeram, R. et B. Amos. (2015). « Beyond 'Inherit It or Marry It': Exploring How Women Engaged in Sustainable Agriculture Access Farmland ». Rural Sociology, 80,1.

Rieux, A. et Dahache, S. (2008). "S'installer comme agricultrice : sur la socialisation et la formation sexuée en agriculture ». Revue d'Etude en Agriculture et Environnement, 88, 3.

Rosenfeld, R. (1985). Farm Women: Work, Farm, and Family in the US. Chapel Hill, NC, University of NC Press.

Sachs, C. (1983). The Invisible Farmers. Totowa, New Jersey, Rowman and Allanheld.

Trauger, A. (2004). "'Because They Can Do the Work': Women Farmers and Sustainable Agriculture ». Gender, Place \& Culture, 11, 2.

USDA. (2014). «Women Farmers ». 2012 Census of Agriculture Highlights, ACH1212/September 2014.

Wright, W. et Annes, A. 2014. «Farm Women and Agritourism: Representing a New Rurality ». Sociologia Ruralis, 54, 4. 